\title{
Relationship Between Blood Lactate and Oxidative Stress Biomarkers Following Acute Exercise
}

\author{
Richard J. Bloomer ${ }^{*}$ and Bradford J. Cole
}

Cardiorespiratory/Metabolic Laboratory, 161 F Roane Fieldhouse, The University of Memphis, Memphis, TN 38152, USA

\begin{abstract}
We measured blood lactate, protein carbonyls (PC), and malondialdehyde (MDA) before and immediately after exercise (graded exercise treadmill test [GXT], Wingate cycle sprint, barbell squat, and barbell bench press) in active men. Data were analyzed using a 4 (exercise mode) x 2 (time) ANOVA with Tukey post hoc testing, and pairwise correlations were made. An interaction was noted for lactate $(\mathrm{p}=0.009)$, with values increasing for all modes, and higher post exercise for bench press compared to Wingate and squat $(\mathrm{p}<0.05)$. No interaction or exercise mode effect was noted for PC or MDA ( $>0.05)$. However, a time effect was noted for PC $(p=0.02)$, with values increasing from pre to post exercise. A positive correlation was noted between PC and MDA ( $r=0.30 ; p=0.0004)$, however no significant correlations were noted between PC and lactate $(r=0.06 ; p=0.51)$ or between MDA and lactate $(r=0.10 ; p=0.22)$. Blood lactate measured during the immediate post exercise period was not significantly correlated to biomarkers of oxidative stress in active men.
\end{abstract}

\section{INTRODUCTION}

Circulating lactate has been shown to rise to upper levels of $8-20 \mathrm{mmol} \cdot \mathrm{L}^{-1}$ with acute anaerobic exercise, as lactate production from accelerated glycolysis overwhelms lactate utilization and clearance [1]. This 4 to 10 fold increase from resting levels results during and immediately following sprinting exercise and intense resistance exercise [1], in addition to graded exercise such as stress testing [2]. Specifically, lactate rises under conditions of increased glycolytic rate, decreased intramuscular oxygen pressure, and decreased lactate oxidation by the liver and kidneys [3].

Acute exercise also increases biomarkers of oxidative stress. Generation of reactive oxygen and nitrogen species (RONS) via normal metabolic pathways can overwhelm endogenous antioxidants and promote oxidation of proteins, lipids, and nucleic acids [4]. The extent of oxidative damage is commonly measured via quantitative assessment of circulating oxidized molecules, such as protein carbonyls (PC) and lipid peroxidation products such as malondialdehyde (MDA) [5]. During intense exercise, excessive RONS production via increased oxygen consumption and cellular metabolism, tissue ischemia/reperfusion, catecholamine autoxidation, as well as activation of RONS generating enzymes (e.g., xanthine oxidase, NADPH oxidase) may contribute to the rise in both $\mathrm{PC}$ and MDA [6].

Previous studies have measured the rise in both lactate and biomarkers of oxidative stress following sprint or resistance exercise [7-11]. Groussard et al. [7] suggest that exercise-induced metabolic acidosis contributes to oxidative

*Address correspondence to this author at the Cardiorespiratory/Metabolic Laboratory, 161F Roane Fieldhouse, The University of Memphis, Memphis, TN 38152, USA; Tel: 901-678-4341; Fax: 901-678-3591;

E-mail: rbloomer@memphis.edu stress, citing the earlier findings of Siesjo et al. [12]. While it is clear that circulating levels of both lactate and oxidative stress biomarkers rise with acute exercise, it is unknown whether the relationship between lactate and RONS (and subsequent elevation in oxidative stress biomarkers) is causative or merely coincidental.

Regarding oxidative stress, Groussard et al. [13] have shown that lactate ion is actually a scavenger of free radicals (hydroxyl radical and superoxide anion) in vitro. Yet, the authors caution that formation of lactate in vivo cannot be dissociated from subsequent metabolic acidosis, which may have a pro-oxidant effect. In vitro models of lactic acidosis have demonstrated generation of free radicals and lipid peroxidation $[12,14,15]$. Yet one must use caution when applying these findings to the matter of human exercise, in particular when considering that the extent of acidosis may not be as extreme within an in vivo system [16-18], and that multiple antioxidants exist within the human blood to minimize the extent of oxidation [4].

Currently, there is controversy regarding the contribution of lactate to exercise induced metabolic acidosis. Robergs et al. propose that glycolysis yields lactate $\left(\mathrm{La}^{-}\right)$, not lactic acid (HLa), and explain that the generation of lactate actually attenuates intracellular acidosis [19,20]. Yet proponents of the traditional concept of lactic acidosis question the veracity of Roberg's stoichiometric calculations. They explain, among other criticisms, that the calculations do not recognize the falling intramuscular $\mathrm{pH}$ of exercising muscle [21-23]. Regardless of the stoichiometric methods used for intramuscular acid/base accounting, $80-100 \%$ of lactate escapes the sarcolemma via facilitated transport or diffusion as lactic acid, effectively resulting in blood lactic acidosis [24].

To date, only Lovlin et al. [11] have specifically analyzed a correlation between lactate formation and oxidative stress. They measured oxidative stress via the 
thiobarbituric acid reactive substances (TBARS) assay, as well as blood lactate in a small sample of six, healthy young men at rest, $40 \% \mathrm{VO}_{2 \max }, 70 \% \mathrm{VO}_{2 \max }$, and $100 \% \mathrm{VO}_{2 \max }$ on a cycle ergometer. While lactate markedly increased with exercise, TBARS was significantly lower at $40 \% \mathrm{VO}_{2 \max }$ and higher at $100 \% \mathrm{VO}_{2 \max }$. The authors explain that exercise at $40 \% \mathrm{VO}_{2 \max }$ enhances antioxidant capacity, yet maximal exercise significantly increases blood lactate and plasma MDA. Overall, Lovlin et al. [11] found a correlation between lactate and MDA $\left(\mathrm{r}^{2}=0.51, \mathrm{p}<0.001\right)$.

The purpose of the current study was to extend the findings of Lovlin and colleagues [11], by using a larger sample size, and measuring blood lactate and oxidative stress biomarkers in response to acute anaerobic exercise in order to determine if correlations exist between these variables.

\section{MATERIALS AND METHODOLOGY}

\section{Subjects}

Data collected from 56 recreationally active men were used in this study. These data are one component of larger data sets associated with studies for which manuscripts have previously been published $[8,25,26]$, inclusive of other variables. Subjects completed a detailed health history questionnaire and underwent a physical examination, including anthropometric testing, prior to enrollment. All subjects were healthy and recreationally active, regularly involved in resistance and aerobic exercise. Subjects did not use medications or nutritional supplements (e.g., antioxidants) that may have affected the dependent variables being measured. All experimental procedures were performed in accordance with the Helsinki Declaration and approved by the University Human Subjects Review Board. Subjects provided both verbal and written consent prior to participating. Subject characteristics are presented in Table 1.

Table1. Characteristics of 56 Recreationally Active Men

\begin{tabular}{|l|c|}
\hline \multicolumn{1}{|c|}{ Variable } & Value \\
\hline \hline Age $(\mathrm{yrs})$ & $24 \pm 5$ \\
\hline Height $(\mathrm{cm})$ & $176 \pm 8$ \\
\hline Weight $(\mathrm{kg})$ & $86 \pm 16$ \\
\hline Body fat $(\%)$ & $14 \pm 6$ \\
\hline Resting Heart Rate $(\mathrm{bpm})$ & $68 \pm 5$ \\
\hline Resting SBP $(\mathrm{mmHg})$ & $120 \pm 9$ \\
\hline Resting DBP $(\mathrm{mmHg})$ & $77 \pm 10$ \\
\hline Hours per week exercise & $4 \pm 3$ \\
\hline Note: Values are mean \pm SD. & \\
\hline
\end{tabular}

\section{Exercise Protocols}

After completion of all screening procedures, a familiarization session for the relevant exercise mode was completed. Then, on a separate day, the following acute bouts were performed: 1) graded exercise treadmill test using the Bruce treadmill protocol (GXT; $n=15)$, Wingate cycle sprint $(n=13)$, barbell squat $(n=13)$, or barbell bench press $(\mathrm{n}=28)$. Please note that the Wingate cycle sprint and the barbell squat were performed by the same subjects on two separate days. For all exercise modes, subjects reported to the laboratory in a fasted (4+ hour) state. They were instructed not to perform any strenuous physical tasks during the 48-hour period preceding the exercise bout. For the GXT, the Bruce treadmill protocol was performed on a motorized treadmill until exhaustion. The average duration of the GXT was 10 minutes. Further details are provided elsewhere [26]. For the Wingate cycle sprint test, subjects pedaled on a Monark friction braked cycle ergometer modified to conduct Wingate testing at a load equal to $7 \%$ of body weight. Prior to the 30 -second test, subjects performed a warm-up of light cycling exercise interspersed with 5second sprints. For the barbell squat, subjects performed 15 repetitions using a load equal to $70 \%$ of subjects' predetermined 1 repetition maximum (1RM) + body weight (system mass), using a Smith machine. Further details for the Wingate and squat test are provided elsewhere [8]. For the barbell bench press, subjects performed 10 sets of 10 eccentric repetitions using a load equal to $70 \%$ concentric 1RM using a Smith machine. Further details are provided elsewhere [25]. For all exercise modes, subjects were instructed and encouraged to exercise until exhaustion, and heart rate (HR) and perceived exertion (RPE) were measured before and immediately after the exercise bout.

\section{Blood Collection and Analysis}

Venous blood samples $(\sim 10 \mathrm{~mL})$ were obtained via needle and collection tube, before and within one minute following exercise. Samples were immediately analyzed for whole blood lactate as an indication of anaerobic metabolism and participant effort (Accutrend; Roche Diagnostics, Mannheim, Germany). The remainder of whole blood was separated immediately to plasma and stored in multiple aliquots at $-80^{\circ} \mathrm{C}$ to be used for the measurement of protein carbonyls (PC) and malondialdehyde (MDA).

Protein carbonyls were analyzed using an enzyme linked immunosorbent assay (ELISA) (Zentech Technology, Dunedin, New Zealand) following the procedures as outlined by the manufacturer. Malondiadehyde was analyzed using a commercially available colorimetric assay (Northwest Life Science Specialties, Vancouver, WA), using the modified method described by Jentzsch and coworkers [27]. Assays were performed in duplicate on first thaw.

\section{Statistical Analysis}

Data were analyzed using a 4 (exercise mode) $\times 2$ (time/intervention) analysis of variance (ANOVA). Tukey post hoc testing was done as needed. Multivariate, pairwise correlations were made between all biochemical variables, in addition to heart rate and RPE. All analyses were performed using JMP statistical software version 4.0 (SAS Institute, Cary, NC). Statistical significance was set at $p<0.05$. The data are presented as mean \pm SEM, except for subject characteristics which are presented as mean \pm SD.

\section{RESULTS}

An exercise mode by time interaction was noted for HR $(p=0.002)$, RPE $(p=0.0002)$, and blood lactate $(p=0.009)$, with values increasing for all variables from pre to post exercise for all modes. Heart rate was higher post exercise for the GXT compared to bench press $(\mathrm{p}<0.05)$, RPE was 
higher post exercise for the GXT compared to bench press and squat $(\mathrm{p}<0.05)$, and lactate was higher post exercise for bench press compared to Wingate and squat $(\mathrm{p}<0.05)$. No interaction or exercise mode effect was noted for $\mathrm{PC}$ or MDA ( $>0.05)$. A time effect was noted for PC $(p=0.02)$, with values increasing from pre to post exercise. However, no time effect was noted for MDA $(p=0.28)$. Data for all exercise modes, pre and post exercise are presented in Table 2.

A positive correlation was noted between HR and RPE ( $\mathrm{r}$ $=0.97 ; \mathrm{p}<0.0001)$, HR and lactate $(\mathrm{r}=0.73 ; \mathrm{p}<0.0001)$, HR and PC $(\mathrm{r}=0.25 ; \mathrm{p}=0.003)$, RPE and lactate $(\mathrm{r}=0.71$; $\mathrm{p}<0.0001)$, RPE and PC $(\mathrm{r}=0.25 ; \mathrm{p}=0.003)$, and PC and MDA $(r=0.30 ; p=0.0004)$. However, no significant correlations were noted between PC and lactate $(\mathrm{r}=0.06 ; \mathrm{p}=$ $0.51)$ or between MDA and lactate $(r=0.10 ; p=0.22)$. When analyzing correlations for each exercise mode independently, it was noted that no differences existed as compared to when data from the four exercise modes was pooled. Therefore, only pooled data are included here. A correlation matrix for all variables is presented in Table 3 .

\section{DISCUSSION}

In the present analysis, differences in blood lactate and oxidative stress biomarkers were noted between the exercise modes (see Table 2); this may be attributed to differences in exercise intensity, mechanics, and duration. Of the exercise modes, the GXT produced the greatest increase in HR $(196.60 \pm 6.79 \mathrm{bpm})$ and RPE $(18.85 \pm 0.65)$, as the GTX likely requires the largest whole body energy expenditure [28]. The bench press produced the lowest HR $(162.21 \pm 4.80 \mathrm{bpm})$ and RPE (14.93 \pm 0.46$)$. This may be expected, because the bench press exercise involved pure eccentric muscle actions and had a relatively limited cardiovascular demand. However, the bench press exercise did produce the greatest increase in lactate from $1.52 \pm 0.35 \mathrm{mmol} \cdot \mathrm{L}^{-1}$ to $8.71 \pm 0.34 \mathrm{mmol} \cdot \mathrm{L}^{-1}$. Additionally, the venous blood samples were obtained from the median cubital vein, which may have a higher concentration of lactate immediately following upper body compared to lower body exercise. Unlike the other exercise modes involving larger muscle groups and/or whole body movement (squats, Wingate, GXT), the bench press alone failed to cause a statistically significant difference in pre and post PC. This may suggest that formation of PC is more closely associated with exercise that challenges larger muscle groups and/or the cardiovascular system.

The strong correlation between lactate, HR, and RPE across all exercise modes is expected. The Borg scale quantifying RPE has been validated as a measure of exertion for both lower extremity and upper extremity exercise [29]. Exertion correlates well with $\mathrm{HR}$ and blood lactate concentration [30].

Interestingly, there was no relationship noted between lactate and PC or lactate and MDA. This is contradictory to the work of Lovlin and colleagues who found an association between MDA and lactate at maximal exertion [11]. The current study used a more specific MDA analysis technique than that of Lovlin et al. [11], which utilized thiobarbituric acid (TBA), a reagent that reacts with other molecules not associated with oxidative stress and has been criticized for its lack of specificity [5]. Additionally, blood was drawn for the current study within one minute of exercise completion, whereas samples were collected at the midpoint of a 5 minute rest period between each stage by Lovlin et al. [11]. Additionally, Lovlin et al. [11] selected a range of exercise intensity from $40-100 \% \mathrm{VO}_{2 \max }$, which differed from the specific exercise performed in the present study. With such a range of intensity, Lovlin et al. [11] reported a much wider range and peak (up to approximately $19 \mathrm{mmol} \cdot \mathrm{L}^{-1}$ ) for blood lactate values, as opposed to our values of only approximately $6-9 \mathrm{mmol} \cdot \mathrm{L}^{-1}$ (Table 2). All of the above mentioned disparities may have contributed to the opposing findings between our work and that of Lovlin and colleagues [11].

The current study does not support the application of in vitro findings by Siesjo et al. [12], Bralet et al. [14], or Fauconneau et al. [15] to explain exercise-induced oxidative stress. These respective investigations involved in vitro models of acidosis at lower $\mathrm{pH}$ levels than that found with anaerobic exercise. The models of Siesjo, Bralet, and Fauconneau and colleagues involved $\mathrm{pH}$ levels of 6.0-6.5 [12], 5.0-6.0 [14] and 3.0 [15], respectively. In an investigation of maximal human exercise, Hermansen et al. [31] found that whole blood $\mathrm{pH}$ dropped from 7.42 to an average low level of 7.11 following a single bout of maximal exercise. The corresponding intramuscular $\mathrm{pH}$ fell from an average of 6.92 to $6.41 \pm 0.04$. Hence, the extent of exercise-

Table 2. Heart Rate, Perceived Exertion, Lactate, Protein Carbonyls, and Molondialdehyde Before (Pre) and After (Post) an Acute Bout of Exercise in Recreationally Active Men

\begin{tabular}{|c|c|c|c|c|c|c|c|c|c|c|}
\hline $\begin{array}{l}\text { Exercise } \\
\text { Mode }\end{array}$ & $\begin{array}{c}\text { HR } \\
\text { Pre } \\
\text { (bpm) }\end{array}$ & $\begin{array}{c}{ }^{*} \mathrm{HR} \\
\text { Post } \\
\text { (bpm) }\end{array}$ & $\begin{array}{c}\text { RPE } \\
\text { Pre } \\
(6-20 \\
\text { Scale })\end{array}$ & $\begin{array}{c}* \text { RPE } \\
\text { Post } \\
(6-20 \\
\text { Scale) }\end{array}$ & $\begin{array}{c}\text { Lactate } \\
\text { Pre } \\
\left(\mathbf{m m o l} \cdot \mathbf{L}^{-1}\right)\end{array}$ & $\begin{array}{c}* \text { Lactate } \\
\text { Post } \\
\left(\mathrm{mmol} \cdot \mathrm{L}^{-1}\right)\end{array}$ & $\begin{array}{c}\text { PC } \\
\text { Pre } \\
(\text { nmol·mg } \\
\left.\text { Protein }^{-1}\right)\end{array}$ & $\begin{array}{c}* * \text { PC } \\
\text { Post } \\
(\text { nmol·mg } \\
\text { Protein }^{-1} \text { ) }\end{array}$ & $\begin{array}{c}\text { MDA } \\
\text { Pre } \\
\left(\mu \mathrm{mol} \cdot \mathbf{L}^{-1}\right)\end{array}$ & $\begin{array}{c}\text { MDA } \\
\text { Post } \\
\left(\mu \mathrm{mol} \cdot \mathrm{L}^{-1}\right)\end{array}$ \\
\hline GXT & $66.10 \pm 6.79$ & $196.60 \pm 6.79$ & $6.00 \pm 0.00$ & $18.85 \pm 0.65$ & $1.57 \pm 0.48$ & $7.94 \pm 0.48$ & $0.11 \pm 0.02$ & $0.14 \pm 0.03$ & $0.74 \pm 0.08$ & $0.75 \pm 0.08$ \\
\hline Sprint & $66.72 \pm 7.66$ & $180.00 \pm 7.33$ & $6.00 \pm 0.00$ & $16.75 \pm 0.71$ & $1.37 \pm 0.54$ & $6.35 \pm 0.53$ & $0.12 \pm 0.03$ & $0.17 \pm 0.03$ & $0.85 \pm 0.09$ & $0.87 \pm 0.09$ \\
\hline Squat & $63.31 \pm 7.04$ & $168.85 \pm 7.04$ & $6.00 \pm 0.00$ & $15.30 \pm 0.67$ & $1.38 \pm 0.51$ & $5.88 \pm 0.50$ & $0.12 \pm 0.02$ & $0.18 \pm 0.03$ & $0.88 \pm 0.08$ & $0.87 \pm 0.08$ \\
\hline Bench Press & $67.12 \pm 4.80$ & $162.21 \pm 4.80$ & $6.00 \pm 0.00$ & $14.93 \pm 0.46$ & $1.52 \pm 0.35$ & $8.71 \pm 0.34$ & $0.10 \pm 0.02$ & $0.11 \pm 0.02$ & $0.76 \pm 0.06$ & $0.74 \pm 0.06$ \\
\hline
\end{tabular}

Note: Values are mean \pm SEM.

Interaction for HR $(p=0.002)$, RPE $(p=0.0002)$, and blood lactate $(p=0.009)$; with values increasing for all variables from pre to post exercise for all modes; *HR higher post exercise for GXT compared to bench press $(\mathrm{p}<0.05)$; *RPE higher post exercise for GXT compared to bench press and squat $(\mathrm{p}<0.05)$; $*$ Lactate higher post exercise for bench press compared to Wingate sprint and squat $(\mathrm{p}<0.05)$; No interaction or exercise mode effect for PC or MDA $(\mathrm{p}>0.05)$; **Time effect noted for PC $(\mathrm{p}=0.02)$.

$\mathrm{GXT}=$ graded exercise treadmill test $($ Bruce treadmill protocol); Sprint $=30$ second Wingate cycle sprint. 
Table 3. Correlation Matrix for Heart Rate, Perceived Exertion, Lactate, Protein Carbonyls, and Molondialdehyde for Data Obtained Before and After an Acute Bout of Exercise in Recreationally Active Men

\begin{tabular}{|c|c|c|c|c|c|}
\hline & Lactate & HR & RPE & PC & MDA \\
\hline \hline Lactate & & $r=0.73 ; p<0.0001$ & $r=0.71 ; p<0.0001$ & $r=0.06 ; p=0.51$ & $r=0.10 ; p=0.22$ \\
\hline HR & $r=0.73 ; p<0.0001$ & & $r=0.97 ; p<0.0001$ & $r=0.25 ; p=0.003$ & $r=0.02 ; p=0.81$ \\
\hline RPE & $r=0.71 ; p<0.0001$ & $r=0.97 ; p<0.0001$ & & $r=0.25 ; p=0.003$ & $r=0.03 ; p=0.69$ \\
\hline PC & $r=0.06 ; p=0.51$ & $r=0.25 ; p=0.003$ & $r=0.25 ; p=0.003$ & & $r=0.30 ; p=0.0004$ \\
\hline MDA & $r=0.10 ; p=0.22$ & $r=0.02 ; p=0.81$ & $r=0.03 ; p=0.69$ & $r=0.30 ; p=0.0004$ & \\
\hline
\end{tabular}

induced whole blood or intramuscular acidosis may not be sufficiently intense to induce lipid peroxidation or protein oxidation, as represented by increased MDA and PC, respectively. This notion supports our finding of no correlation between blood lactate and oxidative stress response to acute exercise.

\section{CONCLUSION}

Evidence clearly supports an increase in blood lactate with acute exercise, with mixed findings for markers of oxidative stress [5]. While in vitro models of lactic acidosis have demonstrated the prooxidant capacity of lactate at high concentrations, there is not sufficient evidence to assume that either blood lactate or intramuscular lactate at exerciseinduced concentrations is associated with oxidative stress, in particular in healthy, trained men. Physiological buffers, antioxidant capacity, and the hemodynamics of human exercise lessen application of the findings of Siesjo et al. [12], which imply a correlation between lactate and exerciseinduced oxidative stress. However, it is possible that untrained subjects, who generally have lower antioxidant capacity, may also clear lactate at a slower rate and thus, present with a greater metabolic acidosis and subsequent susceptibility to oxidative damage.

\section{ACKNOWLEDGEMENT}

This work was supported in part by The University of Memphis Faculty Research Grant Fund. This support does not necessarily imply endorsement by the University of research conclusions.

\section{REFERENCES}

[1] Cairns SP. Lactic acid and exercise performance- culprit or friend? Sports Med 2006; 36: 279-91

[2] Bloomer RB, Creasy AK, Smith WA. Physical work-induced oxidative stress is exacerbated in young cigarette smokers. Nicotine Tob Res 2007; 9: 205-11.

[3] Gladden LB. Lactate metabolism: a new paradigm for the third millennium. J Physiol 2004; 558: 5-30.

[4] Bloomer RJ, Goldfarb AH. Anaerobic exercise and oxidative stress: a review. Can J Appl Physiol 2004; 29: 245-63.

[5] Fisher-Wellman K, Bloomer RJ. Acute exercise and oxidative stress: a 30 year history. Dyn Med 2009; 8: 1 .

[6] Vollaard NB, Shearman JP, Cooper CE. Exercise-induced oxidative stress: myths, realities and physiological relevance. Sports Med 2005; 35: 1045-62.

[7] Groussard C, Rannou-Bekono F, Machefer G, et al. Changes in blood lipid peroxidation markers and antioxidants after a single sprint anaerobic exercise. Eur J Appl Physiol 2003; 89: 14-20.

[8] Bloomer RJ, Fry AC, Falvo MJ, Moore CA. Protein carbonyls are acutely elevated following single set anaerobic exercise in resistance trained men. J Sci Med Sport 2007; 10: 411-17.
[9] Bloomer RJ, Falvo MJ, Fry AC, Schilling BK, Smith WA, Moore CA. Oxidative stress response in trained men following repeated squats or sprints. Med Sci Sports Exerc 2006; 38: 1436-42.

[10] Hudson MB, Hosick PA, McCaulley GO, et al. The effect of resistance exercise on humoral markers of oxidative stress. Med Sci Sports Exerc 2008; 40: 542-8.

[11] Lovlin R, Cottle W, Pyke I, Kavanagh M, Belcastro AN. Are indices of free radical damage related to exercise intensity. Eur J Appl Physiol 1987; 56: 313-6.

[12] Siesjo BK, Bendek G, Koide T, Westerberg E, Wieloch T. Influence of acidosis on lipid peroxidation in brain tissues in vitro. J Cerebral Blood Flow Metab 1985; 5(2): 253-8.

[13] Groussard C, Morel I, Chevanne M, Monnier M, Cillard J, Delamarche A. Free radical scavenging and antioxidant effects of lactate ion: an in vitro study. J Appl Physiol 2000; 89: 169-75.

[14] Bralet J, Bouvier C, Schrieber L, Boquillon M. Effect of acidosis on lipid peroxidation in brain slices. Brain Res 1991; 539: 175-7.

[15] Fauconneau B, Tallineau C, Huguet F, Guillard O, Piriou A. Ironand lactic acid-induced lipid peroxidation in rat kidney homogenates and slices. Biochem Mol Biol Int 1993; 31: 421-7.

[16] Sahlin K, Alvestrand A, Brandt R, Hultman E. Intracellular pH and bicarbonate concentration in human muscle during recovery from exercise. J Appl Physiol Respir Environ Exerc Physiol 1978; 45: 474-80.

[17] Sahlin K, Harris RC, Nylind B, Hultman E. Lactate content and pH in muscle samples obtained afterdymanic exercise. Pflugers Arch 1976; 367: 143-9.

[18] Hermansen L, Onses J. Blood and muscle $\mathrm{pH}$ after maximal exercise in man. J Appl Physiol 1972; 32: 304-8.

[19] Robergs RA, Ghiasvand F, Parker D. Biochemistry of exerciseinduced metabolic acidosis. Am J Physiol Regul Integr Comp Physiol 2004; 287: 502-16.

[20] Robergs RA, Ghiasvand F, Parker D. Lingering construct of lactic acidosis. Am J Physiol Regul Integr Comp Physiol 2005; 289: 904 10 .

[21] Boning D, Strobel G, Beneke R, Maassen N. Lactic acid still remains the real cause of exercise-induced metabolic acidosis. Am J Physiol Regul Integr Comp Physiol 2005; 289: 902-3.

[22] Kemp G, Böning D, Beneke R, Maassen N. Explaining pH change in exercising muscle: lactic acid, proton consumption, and buffering $v s$ strong ion difference. Am J Physiol Regul Integr Comp Physiol 2006; 291: 235-7.

[23] Kemp G. Lactate accumulation, proton buffering, and $\mathrm{pH}$ change in ischemically exercising muscle. Am J Physiol Regul Integr Comp Physiol 2005; 289: 895-901.

[24] Poole RC, Halestrap AP. Transport of lactate and other monocarboxylates across mammalian plasma membranes. Am J Physiol 1993; 264: 761-82.

[25] Bloomer RJ, Falvo MJ, Schilling BK, Smith WA. Prior exercise and antioxidant supplementation: effect on oxidative stress and muscle injury. J Int Soc Sports Nutr 2007; 4: 9.

[26] Bloomer RJ, Creasy AK, Smith WA. Physical work-induced oxidative stress is exacerbated in young cigarette smokers. Nicotine Tob Res 2007; 9: 205-11.

[27] Jentzsch AM, Bachmann H, Fürst P, Biesalski HK. Improved analysis of malondialdehyde in human body fluids. Free Radic Biol Med 1996; 20: 251-6.

[28] Moyna NM, Robertson RJ, Meckes CL, et al. Intermodal comparison of energy expenditure at exercise intensities 
corresponding to the perceptual preference range. Med Sci Sports Exerc 2001; 33: 1404-10.

[29] Borg G, Hassmon P, Lagerstrom M. Perceived exertion related to heart rate and blood lactate during arm and leg exercise. Eur J Appl Physiol 1987; 65: 679-85.
[30] Borg G. Perceived exertion as an indicator of somatic stress. Scand J Rehabil Med 1970; 2: 92-8.

[31] Hermansen L, Onses J. Blood and muscle $\mathrm{pH}$ after maximal exercise in man. J Appl Physiol 1972; 32: 304-8.

(C) Bloomer and Cole; Licensee Bentham Open.

This is an open access article licensed under the terms of the Creative Commons Attribution Non-Commercial License (http://creativecommons.org/licenses/by$\mathrm{nc} / 3.0 /$ /) which permits unrestricted, non-commercial use, distribution and reproduction in any medium, provided the work is properly cited. 